\title{
GENERALIZED HEWITT-SAVAGE THEOREMS FOR STRICTLY STATIONARY PROCESSES
}

\author{
RICHARD ISAAC
}

\begin{abstract}
Generalizations of the Hewitt-Savage zero-one law are proved for strictly stationary processes. This takes the form of statements concerning inclusion and equality relationships among certain sigma-fields related to the process.
\end{abstract}

1. Let $\left\{X_{n}, n \geqslant 1\right\}$ be a sequence of real-valued random variables on the probability space $\left(\Re^{\infty}, \mathscr{B}^{\infty}, P\right)$ where $\Re^{\infty}$ and $\mathscr{B}^{\infty}$ are the usual product space and product Borel $\sigma$-field, respectively. $X_{n}$ may be considered coordinate variables [1]. For $\omega \in \Re^{\infty}$, we denote the $k$ th coordinate of $\omega$ by “ $(\omega)_{k}$ ", so that if $\omega=\left(x_{1}, x_{2}, \ldots\right)$, then $(\omega)_{k}=x_{k}$. Let $N$ be a finite subset of the positive integers $J$, and let $\sigma$ be a permutation of $N$. $\sigma$ defines a function on $\Re^{\infty}$ onto itself by $(\sigma \omega)_{k}=(\omega)_{k \sigma}, k \geqslant 1(k \sigma=k, k \notin N)$. It is easy to see that $\sigma$ is a measurable map, i.e., $\sigma^{-1} A \in \mathscr{B}^{\infty}$ for each $A \in \mathscr{B}^{\infty}$. Let $\Sigma$ be the class of all permutations of every finite subset of $J$, that is, $\Sigma$ is the class of all such $\sigma$ as defined above. An event $A$ is called exchangeable (or symmetrically dependent) if $\sigma^{-1} A=A$ for all $\sigma \in \Sigma$. Actually, we want a slightly larger class of exchangeable events, namely, those for which the relation $\sigma^{-1} A=$ $A(P)$ holds. Here, and hereafter, such a notation means that the relation is understood to hold modulo $P$-null sets. We define $\sigma$ on functions by $(\sigma f)(\omega)$ $=f(\sigma \omega)$.

The exchangeable $\sigma$-field $\mathcal{E}$ is the smallest $\sigma$-field containing the exchangeable events; the tail $\sigma$-field $\mathcal{T}$ is the class of events expressible in terms of $X_{n}$, $X_{n+1}, \ldots$ for any arbitrarily large chosen $n$; the invariant $\sigma$-field $G$ is the class of events such that $T^{-1} A=A$, where $T$ is the usual shift transformation $(T \omega)_{k}=(\omega)_{k+1}$. As with $\mathcal{E}$, the definitions of $\mathcal{T}$ and $\mathcal{G}$ are to be understood modulo $P$.

The Hewitt-Savage 0-1 law [2] asserts that for independent, identically, distributed $X_{n}, \mathcal{E}$ is trivial, that is, $\mathcal{E}$ consists only of events of probability 0 or 1 . In this note we prove generalizations of this result for strictly stationary processes (the process is strictly stationary if $P\left(T^{-1} A\right)=P(A)$ for all events A):

THEOREM 1. If the $X_{n}$ process is strictly stationary, and if the measures $P_{n}$ (see §3) are uniformly absolutely continuous with respect to $P$, then,

Received by the editors March 4, 1976 and, in revised form, July 20, 1976.

AMS (MOS) subject classifications (1970). Primary 60G10, 60F20.

๑ American Mathematical Society 1977 


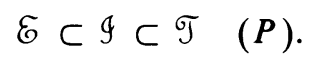

If, in addition, each $\sigma \in \Sigma$ is $P$ nonsingular, i.e., $P\left(\sigma^{-1} A\right)=0$ when $P(A)=0$, there is equality above, that is,

$$
\mathcal{E}=\mathscr{G}=\mathcal{\sigma}(P) .
$$

An easy example serves to show why (2) can be false if the nonsingularity hypothesis is not fulfilled. Consider the two points $\omega_{1}$ and $\omega_{2}$ given by $\left(\omega_{1}\right)_{2 k+1}=1,\left(\omega_{1}\right)_{2 k+2}=0$ for all $k \geqslant 0$, and $\omega_{2}=T \omega_{1}$, and assign probability $\frac{1}{2}$ to each of these points. Let $g=1$ or 0 depending upon whether an infinite number of the even coordinates has entry " 0 " or not, respectively. $g$ is clearly a tail function and not an invariant function. Moreover, in this case $g$ is a.s. equal to a function of $X_{1}$ and $X_{2}$ alone: $g=g_{1}\left(X_{1}, X_{2}\right)(P)$, and $g_{1}$ is $\mathcal{T}$ measurable according to our conventions. However, if $\sigma$ is the permutation interchanging 1 and 2, we obtain

$$
g\left(\omega_{1}\right)=g_{1}(1,0)=1 \neq 0=g_{1}(0,1)=g\left(\omega_{2}\right) \quad(P) .
$$

It follows that $g_{1}$ is not $\mathcal{E}$ measurable, although $\mathcal{T}$ measurable. The trouble here is that $P\left(\left\{\sigma \omega_{1}\right\}\right)=0$ but $P\left(\sigma^{-1}\left\{\sigma \omega_{1}\right\}\right)=\frac{1}{2}$.

Independent identically distributed variables satisfy the nonsingularity condition, and so does the more general class of exchangeable processes. The variables $X_{n}$ are exchangeable if $P\left(\sigma^{-1} A\right)=P(A)$ for all $\sigma \in \Sigma$ and all events $A$. That an exchangeable process is strictly stationary is almost immediate: for finite dimensional cylinder sets exchangeability obviously implies the stationarity relation. Since $P\left(T^{-1} \cdot\right)$ is a measure and $P\left(T^{-1} A\right)=P(A)$ on cylinders, the equality holds for all sets generated by them, namely, all events. Thus (2) holds for exchangeable variables. The relation $\delta=\mathcal{J}$ for exchangeable processes is mentioned in [4, p. 136]. The Hewitt-Savage theorem is a consequence of (2) via the well-known fact that the tail $\sigma$-field of an independent sequence is trivial.

2. Proof of Theorem 1. Because of strict stationarity, it may be assumed that the process is bilateral: $\left\{X_{n},-\infty<n<\infty\right\}$, so that the shift $T$ is a $1-1$ measure preserving point transformation with a $1-1$ measure preserving

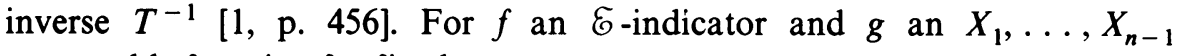
measurable function for fixed $n$, set

$$
A=\left\{\left|f-g\left(X_{1}, \ldots, X_{n-1}\right)\right|>\varepsilon\right\} .
$$

Defining $T_{n}$ (see $\left.\S 3\right)$, observe

$$
\begin{aligned}
T_{n}^{-1} A & =\left\{\left|f-g\left(X_{2}, \ldots, X_{n}\right)\right|>\varepsilon\right\}, \\
T T_{n}^{-1} A & =\left\{\left|T^{-1} f-g\left(X_{1}, \ldots, X_{n-1}\right)\right|>\varepsilon\right\} .
\end{aligned}
$$

Choosing $g$ with $P(A)$ small, $P\left(T T_{n}^{-1} A\right)=P_{n}(A)$ must be small, so $T^{-1} f$ can be approximated by the same functions approximating $f$. Thus $T^{-1} f=f$ or $f=T f$, proving (1). To complete the proof, let $f$ be $\mathcal{T}$ measurable and let $\sigma \in \Sigma$. $f$ differs from a strictly $\mathcal{T}$ measurable function only on a $P$-null set, 
i.e., there is a function $g$ such that $f=g$ a.e. $(P)$ and for each integer $k \geqslant 1$ there is $g_{k}$ depending only upon the coordinates $n \geqslant k$, and $g=g_{k}$ everywhere. Now since $g$ does not depend on any finite initial string of coordinates $\sigma g=g$. Nonsingularity of $\sigma$ easily shows $\sigma f=\sigma g$ a.s. $(P)$ when $f=g$ a.s. $(P)$. Therefore $\sigma f=\sigma g=g=f$ a.s. $(P)$ and so $f$ is $\mathcal{E}$ measurable. The proof is complete.

3. The invariant $\sigma$-field. Let $T_{n}$ be that element of $\Sigma$ defined by: $\left(T_{n} \omega\right)_{k}=$ $(\omega)_{k}$ for $k \geqslant n+1 ;\left(T_{n} \omega\right)_{k}=(\omega)_{k+1}, 1 \leqslant k \leqslant n-1 ;\left(T_{n} \omega\right)_{n}=(\omega)_{1}$. Thus $T_{n} \omega$ and the shift $T \omega$ have the first $n-1$ coordinates identical. For any $\sigma \in \Sigma, \sigma P$ defines a measure by $(\sigma P)(A)=P\left(\sigma^{-1} A\right)$. Set $P_{n}=T_{n} P$ for each $n \geqslant 1$.

TheOREM 2. A necessary and sufficient condition that $\mathcal{E} \subset \mathcal{G}(P)$ is that $P_{n}(C \cap V) \rightarrow P(C \cap V)$ as $n \rightarrow \infty$, for each cylinder set $C$ and each $\mathcal{E}$ set $V$.

Proof. Let $C$ be any cylinder set determined by $X_{1}, X_{2}, \ldots, X_{k}$ for fixed $k \geqslant 1$, and let $f$ be the indicator of an $\delta$-set. If $n \geqslant k+1, T_{n}^{-1} C=T^{-1} C$, and notice that $T f$ is also in $\mathcal{E}$. A standard theorem about transformation of measures [3, p. 163] gives

$$
\int_{C} f d P=\int_{T^{-1} C} T f d P=\int_{T_{n}^{-1} C} T f d P=\int_{C} T f d P_{n}, \quad n \geqslant k+1 .
$$

If the convergence condition above holds, the right side of (4) converges to $\int_{C} T f d P$, and therefore the left side of (4) must equal this quantity. This equality is another way of saying

$$
E\left(T f \mid X_{1}, X_{2}, \ldots, X_{k}\right)=E\left(f \mid X_{1}, X_{2}, \ldots, X_{k}\right),
$$

true for each positive integer $k$. As $k \rightarrow \infty$ the right and left terms of the preceding equality tend to $T f$ and $f$, respectively, by the martingale theorem since both functions are measurable on $X_{1}, X_{2}, \ldots$ Thus $T f=f$ and $f$ is invariant. Conversely, if $f$ in $\delta$ is invariant, the left side of (4) can be written $\int_{C} T f d P$, and then (4) says $P(C \cap V)=P_{n}(C \cap V)$, where $f$ is the indicator of $V$, and $n$ is large enough. This completes the proof of Theorem 2.

4. Concluding remarks. For processes with mutually independent and identically distributed variables or for exchangeable processes, $P_{n}(A)=P(A)$ for all events, and so Theorems 1 and 2 both apply. In the independent case it is well known that $\mathcal{E}, \mathcal{T}$ and $\mathscr{G}$ are all trivial, but in the exchangeable case we obtain $\mathcal{E}=\mathcal{T}=9$ where the equality concerning 9 may be new. The condition of Theorem 2 is a kind of continuity restriction for the transformations $T_{n}^{-1}$ with respect to $T^{-1}$.

\section{REFERENCES}


2. E. Hewitt and L. J. Savage, Symmetric measures on Cartesian products, Trans. Amer. Math. Soc. 80 (1955), 470-501. MR 17, 863.

3. P. R. Halmos, Measure theory, Van Nostrand, Princeton, N.J., 1950. MR 11, 504.

4. J. Neveu, Bases mathématiques du calcul des probabilités, Masson, Paris, 1964. MR 33 \#6659.

Department of Mathematics, lehman College, City University of New York, Bronx, NeW YoRK 10468

Graduate School and University Center, City University of New York, New York, New YoRK 10036 